\title{
NEW RESULTS ON THE ASYMPTOTIC BEHAVIOR OF A THIRD-ORDER NONLINEAR DIFFERENTIAL EQUATION
}

\section{MATHEW OMEIKE}

Abstract. Sufficient conditions are established for the asymptotic behavior of a third-order nonlinear differential equation. Our results improve on Qian's [C. Qian, Asymptotic behavior of a third-order nonlinear differential equation, J. Math. Anal. Appl., 284 (2003), 191-205]

Mathematics subject classification (2010): 34B15, 34C11.

Keywords and phrases: nonlinear differential equation, asymptotic behavior, Lyapunov's method.

\section{REFERENCES}

[1] E. A. BARBAShin, Lyapunov Functions. Izda. 'Nauka', Moscow, 1970.

[2] J. O. C. EZEILO, An elementary proof of a boundedness theorem for a certain third order differential equation, J. Lond. Math. Soc., 38 (1963), 11-16.

[3] J. O. C. EzEILO, Stability results for the solution of some third and fourth order differential equations, Ann. Mat. Pura Appl., 66 (1964), 233-249.

[4] M. GerA, J. R. GRAEF, M. GRegus, On oscillatory and asymptotic properties of solutions of certain nonlinear third order differential equations, Nonlinear Anal., 32 (1998), 417-425.

[5] C. QIAN, On global stability of third-order nonlinear differential equations, Nonlinear Analysis, 42 (2000), 651-661.

[6] C. QIAN, Asymptotic behavior of a third-order nonlinear differential equation, J. Math. Anal. Appl., 284 (2003,) 191-205.

[7] М. О. OMEIKE, Further results on global stability of third-order nonlinear differential equations, Nonlinear Anal., 67 (2007), 3394-3400.

[8] R. Reissig, G. SAnsone, R. Conti, Nonlinear Differential Equations of Higher Order, Noordhoff Inter. Pub. Leyden, 1974.

[9] C. TUNC, On the ultimate boundedness result of the solutions of certain fourth order differential equation, Ann. Differential Equations, 14 (1998), 475-485.

[10] T. Yoshizawa, On the evaluation of the derivatives of solutions of $y^{\prime \prime}=f\left(x, y, y^{\prime}\right)$, Mem. Coll. Sc. Univ. Kyoto, Series A, 28 (1953), 27-32.

[11] T. Yoshizawa, Asymptotic behavior of solutions of a system of differential equations, Contrib. Differential Equations, 1 (1963), 371-387. 